OESOPHAGUS

\title{
5-Aminolevulinic acid photodynamic therapy versus argon plasma coagulation for ablation of Barrett's oesophagus: a randomised trial
}

\author{
M Hage, P D Siersema, H van Dekken, E W Steyerberg, J Haringsma, W van de Vrie, T E Grool, \\ $R$ L P van Veen, H J C M Sterenborg, E J Kuipers
}

Gut 2004;53:785-790. doi: 10.1136/gut.2003.028860

See end of article for authors' affiliations

Correspondence to: Dr P D Siersema, Department of

Gastroenterology and Hepatology, Erasmus MC University Medical Center Rotterdam, PO Box 2040, 3000 CA Rotterdam, the Netherlands; p.siersema@ erasmusmc.n

Accepted for publication 25 November 2003

\begin{abstract}
Background: Photochemical and thermal methods are used for ablating Barrett's oesophagus (BO). The aim of this study was to compare 5-aminolevulinic acid induced photodynamic therapy (ALA-PDT) with argon plasma coagulation (APC) with respect to complete reversal of $\mathrm{BO}$.

Methods: Patients with BO (32 no dysplasia and eight low grade dysplasia) were randomised to one of three treatments: (a) ALA-PDT as a single dose of $100 \mathrm{~J} / \mathrm{cm}^{2}$ at four hours (PDT100; $n=13$ ); (b) ALA-PDT as a fractionated dose of 20 and $100 \mathrm{~J} / \mathrm{cm}^{2}$ at one and four hours, respectively (PDT20+100; $n=13$ ); or (c) APC at a power setting of $65 \mathrm{~W}$ in two sessions (APC; $n=14$ ). If complete elimination of $B O$ was not achieved by the designated treatment, the remaining $\mathrm{BO}$ was treated by a maximum of two sessions of APC.

Results: Mean endoscopic reduction of BO at six weeks was $51 \%$ (range 20-100\%) in the PDT100 group, $86 \%$ (range $0-100 \%$ ) in the PDT20+100 group, and 93\% (range 40-100\%) in the APC group (PDT100 v PDT20+100, $p<0.005$; PDT100 $\vee$ APC, $p<0.005$; and PDT20+100 $\vee$ APC, NS) with histologically complete ablation in 1/13 (8\%) patients in the PDT100 group, 4/12 (33\%) in the PDT20+100 group, and $5 / 14(36 \%)$ in the APC group (NS). Remaining BO was additionally treated with APC in $23 / 40(58 \%)$ patients. Histological examination at 12 months revealed complete ablation in $9 / 11(82 \%)$ patients in the PDT100 group, in $9 / 10(90 \%)$ patients in the PDT20+100 group, and in $8 / 12(67 \%)$ patients in the APC group (NS). At 12 months, no dysplasia was detected. Side effects (that is, pain $(p<0.01)$, and nausea and vomiting $(p<0.05))$ and elevated liver transaminases $(p<0.01)$ were more common after PDT than APC therapy. One patient died three days after treatment with PDT, presumably from cardiac arrhythmia. Conclusion: APC alone or ALA-PDT in combination with APC can lead to complete reversal of Barrett's epithelium in at least two thirds of patients when administered in multiple treatment sessions. As the goal of treatment should be complete reversal of Barrett's epithelium, we do not recommend these techniques for the prophylactic ablation of $\mathrm{BO}$.
\end{abstract}

B arrett's oesophagus (BO) is a premalignant condition in which the normal squamous epithelium of the distal oesophagus is replaced by an incomplete form of intestinal metaplasia, named specialised intestinal metaplasia. ${ }^{1}$ Patients with $\mathrm{BO}$ have an age and sex related risk of $0.5 \%$ per year of developing adenocarcinoma of the oesophagus. $^{2}$

Current management of BO includes endoscopic surveillance at intervals determined by the presence of intestinal metaplasia and the grade of dysplasia within this epithelium. ${ }^{3}$ The rationale for this approach is that surveillance detects high grade dysplasia (HGD) and oesophageal adenocarcinomas at an earlier stage, resulting in a more favourable outcome compared with carcinomas presenting with dysphagia. ${ }^{4}$

A new approach includes the use of endoscopic ablative techniques in combination with antireflux therapy. These techniques can be used therapeutically to remove areas of HGD or early cancer and prophylactically to eliminate the specialised intestinal metaplasia and replace it by squamous epithelium. The currently available ablative modalities are based on photochemical (photodynamic therapy), thermal (argon plasma coagulation, laser, and multipolar electrocoagulation and heater probe), and mechanical (endoscopic mucosal resection) principles.

Photodynamic therapy (PDT) involves light induced activation of an administered photosensitiser which leads to local injury by the production of singlet oxygen. The most commonly used photosensitiser is porfimer sodium (Photofrin). ${ }^{6}$ A novel approach to PDT is endogenous photosensitisation with 5-aminolevulinic acid (ALA). ALA by itself has no photosensitising properties but is metabolised to its photosensitising product protoporphyrin IX. ${ }^{7}$

Argon plasma coagulation (APC) is a non-contact electrocoagulation procedure in which high frequency energy is transmitted to tissue by an ionised gas (argon gas). It induces coagulation of a tissue thickness of up to $2-3 \mathrm{~mm}$, resulting in injury of the superficial layers of tissues. ${ }^{8}$

Currently, all published studies on ablative therapy of BO have dealt with a single treatment modality. In addition, to our knowledge, there are no studies which have compared different techniques of endoscopic reversal of BO. We performed a randomised study comparing the efficacy of single or fractionated dose ALA-PDT with APC for the prophylactic reversal of $\mathrm{BO}$ in 40 patients with $\mathrm{BO}$ without dysplasia or with low grade dysplasia (LGD). If complete elimination of $\mathrm{BO}$ was not achieved by the designated treatment, the remaining BO was ablated by additional APC.

Abbreviations: ALA, 5-aminolevulinic acid; APC, argon plasma coagulation; BO, Barrett's oesophagus; $\mathrm{HGD}$, high grade dysplasia; LGD, low grade dysplasia; PDT, photodynamic therapy; PPls, proton pump inhibitors 
Table 1 Demographic details of patients treated with single dose ALA-PDT (PDT100), fractionated dose ALA-PDT (PDT20+100), or APC

\begin{tabular}{llll}
\hline & PDT100 (n= 13) & PDT20+100 (n=13) & APC (n= 14) \\
\hline Age (y) (median (range)) & $57(52-72)$ & $61(57-69)$ & $60(41-69)$ \\
Sex (M/F) & $10 / 3$ & $10 / 3$ & $11 / 3$ \\
BO length (cm) (median (range)) & $3(2-5)$ & $3(3-4)$ & $3(3-4)$ \\
Histology of BO & 10 & 11 & 1 \\
$\quad$ No dysplasia & 3 & 2 & 3 \\
$\quad$ LGD & $12(9-18)$ & $12(6-24)$ & $12(9-21)$ \\
Follow up in months (median (range)) & $46(40-80)$ & $45(40-80)$ & $51(40-80)$ \\
Mean dose of omeprazole (mg) (median ( & & \\
$\quad$ range)) & & & \\
\hline
\end{tabular}

ALA, 5-aminolevulinic acid; APC, argon plasma coagulation; BO, Barrett's oesophagus; LGD, low grade dysplasia; PDT, photodynamic therapy.

No significant differences between the groups.

\section{PATIENTS AND METHODS Patients}

From January 2001 to July 2002, 40 patients with a BO length of $2-5 \mathrm{~cm}$ were enrolled into the study. Participation was restricted to patients with specialised intestinal metaplasia (Barrett's metaplasia) and no more than LGD on histological examination. Written informed consent was obtained from all patients who were at least 18 years of age. The study was approved by the Institutional Review Board of the Erasmus MC Rotterdam, the Netherlands. All patients were taking proton pump inhibitors (PPIs) for at least six months before treatment. Exclusion criteria were: intolerance to (repeated) endoscopy, pregnancy, acute porphyria, and intercurrent diseases precluding survival during the study period.

\section{Treatment protocol}

All patients underwent baseline endoscopy within two months prior to treatment. The distribution of $\mathrm{BO}$ was recorded and the total length of $\mathrm{BO}$ was measured. Endoscopic photographs were taken at various levels and from different angles in the oesophagus and stored as JPEG files. Four quadrant biopsies were taken at $2 \mathrm{~cm}$ intervals with a standard biopsy forceps. Biopsies were stained with haematoxylin-eosin, and in some cases with alcian blue at $\mathrm{pH}$ 2.5. All biopsies were reviewed by a single experienced gastrointestinal pathologist (HvD). Randomisation was performed by the trial centre of the Department of Internal Oncology, Erasmus MC Rotterdam. Patients were stratified for the presence of no dysplasia or LGD in baseline biopsies.

For ALA-PDT, $60 \mathrm{mg} / \mathrm{kg}$ ALA (Sigma-Aldrich Chemie BV, Zevenaar, the Netherlands) was dissolved in $20 \mathrm{ml}$ of orange juice. All patients were kept in a darkened room for 36 hours after ingestion. Blood cell counts and liver enzymes levels were determined at baseline and the day after treatment. A KTP/532 dye laser module (Laser scope, San Jose, California, USA) delivered light with a wavelength of $630 \mathrm{~nm}$. PDT illumination schemes were: (a) a single laser illumination with a fluence (light dose) of $100 \mathrm{~J} / \mathrm{cm}^{2}$ at four hours after ALA administration (PDT100 group) or (b) a fractionated laser illumination with a fluence of $20 \mathrm{~J} / \mathrm{cm}^{2}$ and $100 \mathrm{~J} / \mathrm{cm}^{2}$ at one and four hours, respectively, after ALA administration (PDT20+100 group). Light delivery was performed using an inflatable balloon with an inflated diameter of $2.5 \mathrm{~cm}$ (Wizzard X-cell; Wilson-Cook Medical, Inc., WinstonSalem, North Carolina, USA). The total output power of the cylindrical diffuser $(400 \mu \mathrm{m}$ fibre core diameter $)$ (CeramOptec GmbH, Bonn, Germany) was measured in an integrating sphere (Grasbery Optronics S370; TeLintelo Systems, the Netherlands) and was set to a value yielding a calculated fluence rate of $100 \mathrm{~mW} / \mathrm{cm}^{2}$. The cylindrical diffuser was aligned in the balloon prior to the procedure.
The length of the window of the balloon and the cylindrical diffuser were either 3 or $5 \mathrm{~cm}$, depending on the estimated length of BO. The deflated balloon was positioned over a guidewire and its position was checked endoscopically. The output power of the cylindrical diffuser was checked prior to and post ALA-PDT.

For APC, an Argon Beamer 2 device, APC 300 (Erbe Medizintechnik, Tübingen, Germany) was used with a gas flow rate of $2 \mathrm{l} / \mathrm{min}$ at a power setting of $65 \mathrm{~W}$. We aimed to ablate two thirds of the oesophageal circumference of BO during the first session while in the following session the remainder of the $\mathrm{BO}$ was ablated. Ablation was initiated at the gastro-oesophageal junction and than proceeded longitudinally towards the proximal squamocolumnar junction. APC comprised a maximum of two treatment sessions per patient at four week intervals.

\section{Follow up protocol}

Patients were treated with a daily dose of at least $40 \mathrm{mg}$ omeprazole (AstraZeneca, Zoetermeer, the Netherlands). Both patients and their general practitioners were instructed that this dose of PPI needed to be continued during the study. Patient symptoms during and after ablative therapy were recorded. In addition, patients were contacted by telephone on day 5 after treatment to assess side effects.

Follow up endoscopies were performed at six weeks and at $6,12,18$, and 24 months after ablative treatment. During

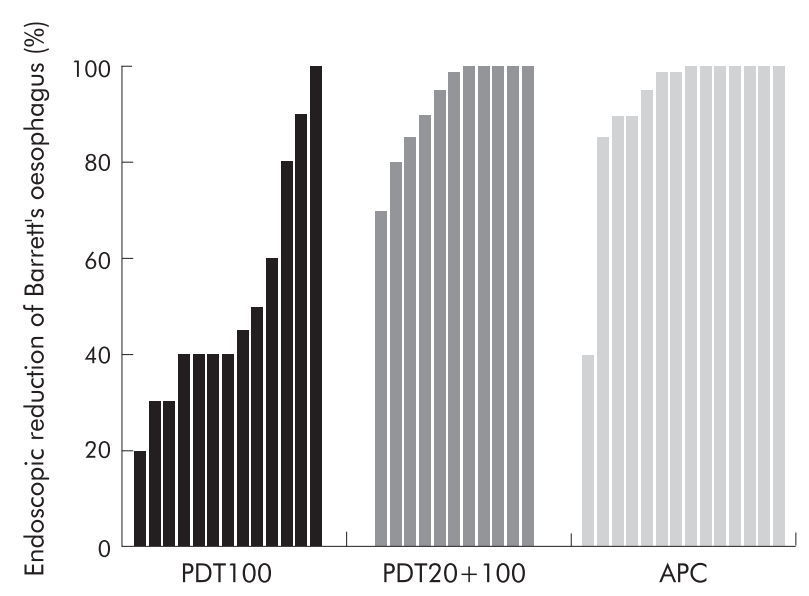

Figure 1 Endoscopic reduction of Barrett's oesophagus at six weeks after treatment, in patients given single dose 5-aminolevulinic acid (ALA)-photodynamic therapy (PDT) (PDT100; $n=13$ ), fractionated dose ALA-PDT (PDT20+100; $n=12$ ), and argon plasma coagulation (APC) $(n=14)$. (PDT100 group $v$ PDT20+100 group, $p<0.005 ;$ PDT100 group $\checkmark$ APC group, $p<0.005$; PDT20+100 group $v$ APC group, NS.) 
Table 2 Microscopic evaluation at six weeks after treatment in patients treated with single dose ALA-PDT (PDT100; $n=13)$, fractionated dose ALA-PDT (PDT20+100; $n=12)$, or APC $(n=14)$

\begin{tabular}{llll}
\hline & No BO & Residual BO & Sub-squamous BO \\
\hline PDT100 & 1 & 12 & 0 \\
PDT20+100 & 4 & 7 & 1 \\
APC & 5 & $4^{*}$ & 5 \\
\hline
\end{tabular}

ALA, 5-aminolevulinic acid; APC, argon plasma coagulation; $B O$ Barrett's oesophagus; PDT, photodynamic therapy. *Two of these patients also had sub-squamous BO. No significant differences between the groups.

follow up endoscopies, photographs were taken and stored as JPEG files. In addition, biopsies were taken from all four quadrants at $2 \mathrm{~cm}$ intervals throughout the BO segment. Endoscopic pictures were assessed by two investigators (PDS, $\mathrm{MH}$ ) who were blinded to the treatment that the patient had received. On histological examination, samples were scored for the presence or absence of specialised intestinal metaplasia and, if present, whether this was found next to or underneath (regenerated) squamous epithelium. In addition, the highest grade of dysplasia was recorded.

If macroscopic BO was observed at the first follow up endoscopy at six weeks after treatment, this BO was ablated by additional APC with a maximum of two sessions at four week intervals.

Results were expressed as: (1) endoscopic reduction of the BO surface and (2) microscopic presence or absence of Barrett's metaplasia at six weeks and at 6, 12, 18, and 24 months after the initial treatment.

\section{Statistical analysis}

Endoscopic reduction of the BO surface was compared between the PDT100 group, PDT20/100 group, and APC group using Mann-Whitney $U$ tests for all three possible comparisons. Adjustment for multiple comparisons was not performed as all three comparisons were considered to be of interest. Patient demographics, histological results, and symptoms after ablative treatment were compared using the $\chi^{2}$ test. A difference was considered significant if $\mathrm{p}<0.05$.

\section{RESULTS}

Forty (31 males/nine females) patients with a mean BO length of $3 \mathrm{~cm}$ (range $2-5)$ and no dysplasia $(\mathrm{n}=32$ ) or with LGD $(\mathrm{n}=8)$ on histological examination were included. Median age of all patients was 59 years (range 41-72). The mean dose of omeprazole after treatment was $47.5 \mathrm{mg}$ (range 40-80). The three patients groups were demographically similar (table 1).
All patients completed the designated ablative therapy. Of the 26 patients treated with ALA-PDT, 24 (96\%) showed endoscopic reduction of $\mathrm{BO}$ at six weeks after treatment (fig 1). One patient treated with a fractionated dose of PDT showed no response and another patient treated with a fractionated dose of PDT died shortly after treatment (see below). Mean endoscopic BO surface reduction was $51 \%$ (range $20-100 \%$ ) in the PDT100 group and $86 \%$ (range $0-100 \%)$ in the PDT20+100 group. No endoscopic evidence of remaining $\mathrm{BO}$ was seen in one $(8 \%)$ patient in the PDT100 group and in five $(42 \%)$ patients in the PDT20+100J group. All 14 patients treated with APC showed endoscopic reduction of $\mathrm{BO}$ at six weeks after treatment. Mean endoscopic regression was $93 \%$ (range $40-100 \%$ ). No endoscopic evidence of remaining BO was seen in seven (50\%) patients in the APC group (PDT100 group $v$ PDT20+100 group, $\mathrm{p}<0.005$; PDT100 group $v$ APC group, $\mathrm{p}<0.005$; PDT20+100 group $v$ APC group, NS).

Histological evaluation at six weeks after treatment revealed complete reversal of $\mathrm{BO}$ in one $(8 \%)$ patient in the PDT100 group, in four (33\%) patients in the PDT20+100 group, and in five (36\%) patients in the APC group (NS). The presence of $\mathrm{BO}$ after treatment was predominantly found as BO next to (regenerated) squamous epithelium in both PDT groups. In the APC group, $\mathrm{BO}$ was found as $\mathrm{BO}$ underneath squamous epithelium (sub-squamous BO) in $7 / 9$ patients (table 2).

All patients with macroscopic $\mathrm{BO}$ at the follow up endoscopy at six weeks received additional APC treatment in one (20 patients) or two (three patients) sessions (fig 2). Follow up endoscopy at six months showed macroscopic BO in one $(7 \%)$ patient in the APC group (table 3) whereas histological evidence of $\mathrm{BO}$ was found in one patient in the PDT100 group and in three patients in the APC group. Endoscopic presence of BO at 12 months was observed in one patient in the PDT100 group and in two (17\%) patients in the APC group. Histological examination at 12 months revealed $\mathrm{BO}$ in two patients in the PDT100 group, in one patient in the PDT20+100 group, and in four patients in the APC group. Endoscopic BO at 18 months was observed in two patients in the PDT100 group and in two patients in the APC group whereas histological evidence of $\mathrm{BO}$ was found in two patients in the PDT100 group, in one patient in the PDT20+100 group, and in three patients in the APC group. During follow up, no dysplasia was observed in any of the biopsies with remaining $\mathrm{BO}$.

Side effects are shown in table 4. Pain during treatment, and nausea and vomiting were more common in patients treated with ALA-PDT compared with APC. One patient treated with APC developed a stricture which was effectively treated by a single session of dilation. Another patient treated with a fractionated dose of ALA-PDT died suddenly

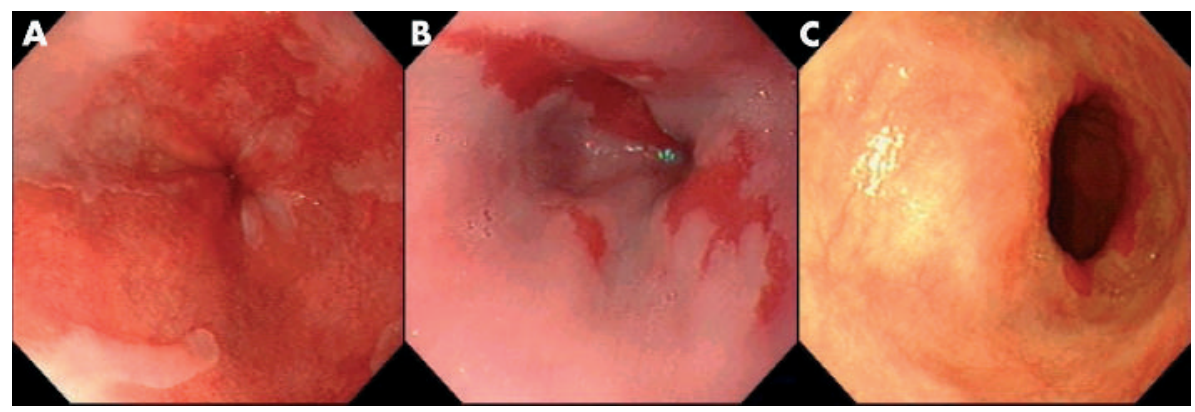

Figure 2 Endoscopic views of a patient with Barrett's oesophagus (BO): (A) before treatment, (B) $80 \%$ reversal of BO at six weeks after treatment with a fractionated dose of 5-aminolevulinic acid-photodynamic therapy, and (C) complete reversal of $\mathrm{BO}$ at six months after additional treatment with argon plasma coagulation. 
Table 3 Barrett's oesophagus during follow up in patients treated with single dose ALAPDT (PDT100), fractionated dose ALA-PDT (PDT20+100), or APC, in some patients followed by treatment with APC for residual Barrett's oesophagus

\begin{tabular}{|c|c|c|c|c|c|c|}
\hline \multirow[b]{2}{*}{$\begin{array}{l}\text { Follow up } \\
\text { (months) }\end{array}$} & \multicolumn{2}{|l|}{ PDT100 group } & \multicolumn{2}{|c|}{ PDT20+100 group } & \multicolumn{2}{|l|}{ APC group } \\
\hline & $\begin{array}{l}\text { Endoscopic BO } \\
(\%)\end{array}$ & $\begin{array}{l}\text { Histological BO } \\
(\%)\end{array}$ & $\begin{array}{l}\text { Endoscopic BO } \\
(\%)\end{array}$ & $\begin{array}{l}\text { Histological BO } \\
(\%)\end{array}$ & $\begin{array}{l}\text { Endoscopic BO } \\
\text { (\%) }\end{array}$ & $\begin{array}{l}\text { Histological BO } \\
(\%)\end{array}$ \\
\hline 6 & $0 / 13$ & $1 / 13(8)$ & $0 / 12$ & $0 / 12$ & $1 / 14(7)$ & $3 / 14(21)$ \\
\hline 12 & $1 / 11$ (9) & $2 / 11$ (18) & $0 / 10$ & $1 / 10(10)$ & $2 / 12$ (17) & 4/12 (33) \\
\hline 18 & $2 / 8(25)$ & $2 / 8(25)$ & $0 / 8$ & $1 / 8(12)$ & $2 / 9(22)$ & $3 / 9(33)$ \\
\hline 24 & NA & NA & $0 / 2$ & $0 / 2$ & NA & NA \\
\hline
\end{tabular}

*NA, not applicable.

ALA, 5-aminolevulinic acid; APC, argon plasma coagulation; BO, Barrett's oesophagus; PDT, photodynamic therapy.

No significant differences between the groups.

three days after treatment. This patient had left hospital one hour earlier and had not reported clinical symptoms at the moment of departure. At autopsy, the oesophageal wall showed microscopic signs of transmural necrosis without perforation. Although an atherosclerotic change of the arterial blood vessels was observed, no evidence of an ischaemic cardiovascular event was found. The autopsy revealed no clear explanation for the sudden death in this patient. Twenty patients treated with ALA-PDT had mild elevation of transaminase levels the day after treatment (aspartate aminotransferase: mean $62 \mathrm{U} / \mathrm{l}$ (range 39-89) (reference 5-30 U/1); alanine transferase: mean $72 \mathrm{U} / \mathrm{l}$ (range 51-116) (reference 10-40 U/1)). At six weeks, liver enzyme levels in all patients had normalised.

\section{DISCUSSION}

This is the first study in which a photochemical method for removing BO (that is, ALA-PDT) was compared with a presently available thermal modality (that is, APC) for prophylactic reversal of Barrett's intestinal metaplasia to squamous epithelium.

Single treatment with ALA-PDT and APC initially reduced the total surface of BO by $96 \%$ and $100 \%$, respectively (fig 1). However, complete elimination of $\mathrm{BO}$ was seen in only five of $25(20 \%)$ patients treated with ALA-PDT alone and in five of $14(36 \%)$ patients treated with two sessions of APC at 6 weeks after treatment (table 2). After subsequent treatment with APC in patients with persistent BO at the first follow up endoscopy at six weeks, complete microscopic reversal was obtained in 18 of $21(86 \%)$ patients in both ALAPDT groups and in eight of $12(67 \%)$ patients in the APC group at 12 months after the initial treatment (table 3 ). In patients with residual $\mathrm{BO}$, no evidence of dysplasia was found.

Table 4 Side effects and complications in patients treated with ALA-PDT (single dose and fractionated dose combined) or APC

\begin{tabular}{lccl}
\hline & PDT $(\mathbf{n}=\mathbf{2 6})$ & APC $(\mathbf{n = 1 4 )}$ & $\mathbf{p ~ V a l u e ~}$ \\
\hline Pain during treatment & 23 & 5 & $<0.01$ \\
Odynophagia & 24 & 12 & $\mathrm{NS}$ \\
Fever & 8 & 2 & $\mathrm{NS}$ \\
Nausea and vomiting & 7 & 0 & $<0.05$ \\
Sudden death & $1^{*}$ & 0 & $\mathrm{NS}$ \\
Stricture formation & 0 & 1 & NS \\
Elevated liver enzyme tests & 20 & 0 & $<0.01$ \\
\hline
\end{tabular}

ALA, 5-aminolevulinic acid; APC, argon plasma coagulation; PDT photodynamic therapy.

*Patient died, presumably from cardiac arrhythmia.
How do our results compare with other studies? Two larger studies on the use of ALA-PDT for BO have been published. One therapeutic study by Gossner and colleagues ${ }^{10}$ reported eradication of HGD in $\mathrm{BO}$ in all 10 patients and elimination of intramucosal cancer in 17 of 22 patients, mostly after two separate ALA-PDT treatments. Squamous re-epithelialisation was observed in only two thirds of patients and was incomplete in all of them. In another study by Ackroyd and colleagues, ${ }^{11} 18$ patients with LGD in BO were treated by one session of ALA-PDT. The median decrease in the surface of BO was 30\%, and none of the control biopsies from remaining Barrett's epithelium showed dysplasia.

We applied two different PDT illumination schemes: (a) a single illumination scheme with an energy dose of $100 \mathrm{~J} / \mathrm{cm}^{2}$ at four hours and (b) a fractionated illumination scheme with an energy dose of $20 \mathrm{~J} / \mathrm{cm}^{2}$ and $100 \mathrm{~J} / \mathrm{cm}^{2}$ at one and four hours, respectively, after ALA ingestion. The latter scheme was chosen because our previous experimental work had shown that a fractionated ALA-PDT scheme increased cell death in human EBV transformed cell lines. We demonstrated that an early course of ALA-PDT inhibited the haeme biosynthetic enzyme ferrochelatase while the activity of another rate limiting enzyme, porphobilinogen deaminase, remained intact. This resulted in increased accumulation of the photosensitiser protoporphyrin IX and increased efficacy of a second course of ALA-PDT. ${ }^{12}$ There is however no agreement in the literature on whether a fractionated illumination scheme enhances the therapeutic effect of PDT. ${ }^{13}{ }^{14}$ In agreement with our experimental results, we found that the fractionated dose of ALA-PDT caused more pronounced regression of $\mathrm{BO}$ at six weeks than the single dose, both macroscopically (fig 1) and microscopically (table 2), although the latter did not reach statistical significance.

The use of APC for reversing $\mathrm{BO}$ has been reported in several studies. ${ }^{15-22}$ In these studies, complete reversal of BO ranged from $42 \%$ to $98 \%$ after $1-6$ treatments with APC. In our study, $67 \%$ of patients had a complete histological response one year after 2-4 treatment sessions with APC (table 3). This somewhat disappointing result can, at least in part, be explained by our strict definition of persistent $\mathrm{BO}$, according to which any biopsy showing specialised columnar epithelium was considered to be persistent BO. Islands of BO underlying newly formed squamous epithelium were found in 7/9 patients with an incomplete response after the initial treatment with APC. Sub-squamous islands of BO were more often found after APC (50\%) than after ALA-PDT (4\%) (table 2) which is in accordance with the findings of others. ${ }^{1021}$ Sub-squamous BO may regress or remain clinically unimportant if protected by the newly formed squamous epithelium from repeated attacks by gastric and duodenal fluid. However, there are reasons to believe that these islands 
retain their malignant potential. At least two cases of adenocarcinoma arising under the newly formed squamous epithelium have been reported after APC. ${ }^{23}{ }^{24}$

It has been suggested that the results of APC can be improved by using a power setting of $65 \mathrm{~W}$ or more. Higher power APC settings may result in deeper tissue destruction and therefore more complete ablation of $\mathrm{BO} .{ }^{17}{ }^{18}$ In addition, recurrence of BO after APC was found to be related to the presence of a long segment of $\mathrm{BO}(>3 \mathrm{~cm})$ and to persistent acid reflux, as measured by $\mathrm{pH}$ monitoring and/or reduction of PPI dose. ${ }^{21} 22$ In our study, we performed APC at a power setting of $65 \mathrm{~W}$, the median length of $\mathrm{BO}$ was $3 \mathrm{~cm}$, and the mean daily dose of omeprazole was $45 \mathrm{mg}$ in the APC treatment group. As oesophageal $\mathrm{pH}$ monitoring was not performed in our study, we cannot exclude the fact that incomplete ablation of $\mathrm{BO}$ may also be explained by higher exposure to $\mathrm{pH}$ levels $<4$ in these patients.

Until a few years ago, the main goal of ablative therapy was histological downgrading of dysplasia. Recent studies have however demonstrated that genetic abnormalities can persist in BO after ablative therapy. ${ }^{25} 26$ In addition, van Hillegersberg and colleagues ${ }^{27}$ recently described two patients who developed adenocarcinoma after incomplete endoscopic ablation of Barrett's epithelium. Therefore, the goal of treatment should be complete elimination of all Barrett's metaplastic tissue. Whenever macroscopic BO was observed at the first follow up endoscopy at six weeks, we treated this with additional APC, resulting in a further increase in the number of patients macroscopically and microscopically free of $\mathrm{BO}$ at 12 months of follow up. However, of the more than $60 \%$ of patients followed up for 18 months, $12-33 \%$ were still found to have $\mathrm{BO}$ next to or underneath the regenerated squamous epithelium (table 3 ). Therefore, the injury produced by ALA-PDT, APC, or a combination of both, was not sufficient to completely reverse $\mathrm{BO}$ in all patients. As BO is thought to originate from cells in the deeper layers of the oesophageal wall, ${ }^{28}$ it may be well that even after complete reversal of Barrett's epithelium, and in the presence of persistent acid reflux, recurrent growth of $\mathrm{BO}$ is more successful than squamous regeneration.

One of the reasons why the photosensitiser ALA has become more popular than Photofrin in Europe is its presumed lower incidence of side effects. We saw no skin photosensitivity after ALA-PDT. In contrast, owing to its long half life, patients are advised to avoid sunlight (UV) for a period of 4-6 weeks after being treated with Photofrin. No strictures were observed in our patients treated with ALAPDT while strictures have been reported in $34 \%$ of patients given Photofrin. ${ }^{6}$ Severe chest pain during treatment was an important side effect of ALA-PDT, necessitating the use of high doses of analgesics (table 4). Another (transient) side effect of ALA-PDT was a two- to threefold increase in transaminase levels, reflecting liver parenchyma damage. Independent of the presence of light, a local autocatalytic interaction between ALA, iron, and oxygen has been demonstrated in the liver which could be responsible for the parenchyma specific increase in liver enzymes. ${ }^{29}$ Finally, an unexpected finding was a sudden death in a patient who was treated with a fractionated dose of ALA-PDT three days before his death. He had no clinical symptoms when leaving hospital, and collapsed one hour after having been discharged. Autopsy showed microscopic signs of transmural necrosis of the oesophageal wall at the site where the treatment had been performed but no evidence of perforation or mediastinal inflammation. In an earlier study, we also observed necrosis of the layers of the oesophageal wall in rats treated with PDT. None of these rats developed perforation of the oesophagus. ${ }^{30}$ A possible explanation for the unexpected death in this patient may have been cardiac arrhythmia.
Atrial fibrillation has been reported during and following oesophageal PDT. ${ }^{31} 32$

In conclusion, APC alone or ALA-PDT in combination with APC can lead to complete reversal of Barrett's epithelium in at least two thirds of patients when administered in multiple treatment sessions. Single modality treatment (ALA-PDT alone or APC in two sessions) resulted in persistence of BO in the majority of patients, even when the immediate posttreatment macroscopic appearance suggested adequate treatment. We were unable to show a significant difference in efficacy between the treatments. Treatment with ALA-PDT was accompanied by more side effects than APC. As the goal of treatment should be complete reversal of Barrett's epithelium to squamous epithelium, we do not recommend these techniques for the prophylactic ablation of BO.

\section{ACKNOWLEDGEMENTS}

M Hage was supported financially by the Revolving Fund of the Erasmus MC University Medical Centre Rotterdam. We thank Mr Jan van de Berg for technical assistance during the PDT treatments.

\section{Authors' affiliations}

M Hage, Department of Gastroenterology and Hepatology, and Department of Pathology, Erasmus MC University Medical Centre Rotterdam, the Netherlands

P D Siersema, J Haringsma, W van de Vrie, T E Grool, E J Kuipers, Department of Gastroenterology and Hepatology, Erasmus MC University Medical Centre Rotterdam, the Netherlands

H van Dekken, Department of Pathology, Erasmus MC University Medical Centre Rotterdam, the Netherlands

E W Steyerberg, Department of Public Health, Erasmus MC University Medical Centre Rotterdam, the Netherlands

R L P van Veen, H J C M Sterenborg, Department of Radiation Oncology (Photodynamic Therapy and Optical Spectroscopy Research Program), Erasmus MC University Medical Center Rotterdam, the Netherlands

\section{REFERENCES}

1 Spechler SJ. Barrett's oesophagus. New Engl J Med 2002;346:836-42.

2 Shaheen NJ, Crosby MA, Bozymski EM, et al. Is there publication bias in the reporting of cancer risk in Barrett's oesophagus? Gastroenterology 1998:83:2049-53.

3 Sampliner RE and the Practice Parameters Committee of the American College of Gastroenterology. Updated guidelines for the diagnosis, surveillance, and therapy of Barrett's oesophagus. Am J Gastroenterol 2002;97:1888-95.

4 van Sandick JW, van Lanschot JJB, Kuiken BW, et al. Impact on endoscopic biopsy surveillance of Barrett's oesophagus on pathological stage and clinical outcome of Barrett's carcinoma. Gut 1998;43:216-22.

5 van den Boogert J, van Hillegersberg R, Siersema PD, et al. Endoscopic ablation therapy for Barrett's oesophagus with high-grade dysplasia: a review. Am J Gastroenterol 1999;94:1153-60.

6 Overholt BF, Panjehpour M, Haydek JM. Photodynamic therapy for Barrett's oesophagus: follow-up in 100 patients. Gastrointest Endosc 1999;49:1-7.

7 Hinnen P, de Rooij FW, Terlouw EM, et al. Porphyrin biosynthesis in human Barrett's ooesophagus and adenocarcinoma after ingestion of 5aminolaevlinic acid. Br J Cancer 2000;83:539-43.

8 Wahab PJ, Mulder CJ, den Hartog G, et al. Argon plasma coagulation in flexible gastrointestinal endoscopy: pilot experiences. Endoscopy 1997;29:176-81

9 van Veen RLP, Aalders MCG, Pasma KL, et al. In-situ light dosimetry during photodynamic therapy of Barrett's esophagus with 5-aminolevulinic acid. Lasers Surg Med 2002;31:299-304.

10 Gossner L, Stolte M, Sroka R, et al. Photodynamic ablation of high-grade dysplasia and early cancer in Barrett's oesophagus by means of 5aminolevulinic acid. Gastroenterology 1998;114:448-55.

11 Ackroyd R, Brown NJ, Davis MF, et al. Photodynamic therapy for dysplastic Barrett's ooesophagus: a prospective, double blind, randomised, placebo controlled trial. Gut 2000;47:612-17.

12 Hinnen $P$, Siersema PD, Edixhoven A, et al. A two course illuminating scheme improves ALA-PDT efficacy. Cell Mol Biol 2002;48:903-9.

13 Dolmans DEJGJ, Kadambi A, Hill JS, et al. Targeting tumor vasculature and cancer cells in orthotopic breast tumor by fractionated photosensitizer dosing photodynamic therapy. Cancer Res 2002;62:4289-94.

14 Babilas P, Schacht V, Liebscg G, et al. Effects of light fractionation and different fluence rates on photodynamic therapy with 5-aminolaevulinic acid in vivo. Br J Cancer 2003;88: 1462-9.

15 van Laethem J-L, Cremer M, Peny MO, et al. Eradication of Barrett's mucosa with argon plasma coagulation and acid suppression: immediate and mid term results. Gut 1998;43:747-51 
16 Mork H, Barth T, Kreipe HH, et al. Reconstitution of squamous epithelium in Barrett's oesophagus with endoscopic argon plasma coagulation: a prospective study. Scand J Gastroenterol 1998;33:1130-4.

17 Pereira-Lima JC, Busnello JV, Saul C, et al. High power setting argon plasma coagulation for the eradication of Barrett's oesophagus. Am J Gastroenterol 2000;95:1661-8.

18 Schulz H, Miehlke S, Antos D, et al. Ablation of Barrett's epithelium by endoscopic argon plasma coagulation in combination with high-dose omeprazole. Gastrointest Endosc 2000;51:659-63.

19 Tigges H, Fuchs KH, Maroske J, et al. Combination of endoscopic argon plasma coagulation and anti-reflux surgery for treatment of Barrett's oesophagus. J Gastrointest Surg 2001;5:251-9.

20 Morris CD, Byrne JP, Armstrong GRA, et al. Prevention of the neoplastic progression of Barrett's oesophagus by endoscopic argon beam plasma coagulation. Br J Surg 2001;88:1357-62.

21 Basu KK, Pick B, Bale R, et al. Efficacy and one year follow up of argon plasma coagulation therapy for ablation of Barrett's ooesophagus: factors determining persistence and recurrence of Barrett's epithelium. Gut 2002;51:776-80.

22 Kahaleh M, van Laethem J-L, Nagy N, et al. Long-term follow-up and factors predictive of recurrence in Barrett's oesophagus treated by argon plasma coagulation and acid suppression. Endoscopy 2002;34:950-5.

23 van Laethem JL, Peny MO, Salmon I, et al. Intramucosal adenocarcinoma arising under squamous re-epithelialisation of Barrett's ooesophagus. Gut 2000;46:574-7.
24 Shand A, Dallal H, Palmer K, et al. Adenocarcinoma arising in columnar lined ooesophagus following treatment with argon plasma coagulation. Gut 2001;48:580-1.

25 Garewal H, Ramsey L, Sharma P, et al. Biomarker studies in reversed Barrett's oesophagus. Am J Gastroenterol 1999:94:2829-33.

26 Krishnadath KK, Wang KK, Taniguchi K, et al. Persistent genetic abnormalities in Barrett's oesophagus after photodynamic therapy. Gastroenterology 2000; 1 19:624-30

27 van Hillegersberg R, Haringsma J, ten Kate FJW, et al. Invasive carcinoma after endoscopic ablative therapy for high-grade dysplasia in Barrett's oesophagus. Dig Surg 2003;20:440-4.

28 Jankowski JA, Harrison RF, Perry I, et al. Barrett's metaplasia. Lancet 2000;356:2079-85.

29 Rocha ME, Dutra F, Bandy B, et al. Oxidative damage to ferritin by 5aminolevulinic acid. Arch Biochem Biophys 2003;409:349-56.

30 van den Boogert J, van Hillegersberg R, van Staveren $\mathrm{HJ}$, et al. Timing of illumination is essential for effective and safe photodynamic therapy: a study in normal rat oesophagus. Br J Cancer 1999;79: 825-30.

31 Wolfsen HC, Woodward TA, Raimondo M. Photodynamic therapy for dysplastic Barrett oesophagus and early oesophageal adenocarcinoma. Mayo Clin Proc 2002;77: 1176-81.

32 Overholt BF, Panjehpour M, Ayres M. Photodynamic therapy for Barrett's oesophagus: cardiac effects. Lasers Surg Med 1997;21:317-20. 